\title{
Effect of Different Soil Water Content and Seed Storage on Quality of Six Range Grasses in the Semi-Arid Ecosystems of Kenya
}

\author{
Koech O. K. ${ }^{1, *}$, Kinuthia R. ${ }^{1}$., Mureithi S. $M^{1}$., Karuku G.. ${ }^{1}$, Wanjogu R. ${ }^{2}$ \\ ${ }^{1}$ Department of Land Resource Management and Agricultural Technology, University of Nairobi, Kenya \\ ${ }^{2}$ National Irrigation Board, Mwea Irrigation Agricultural Development (MIAD) Centre P O Box 210, 10303, WANGURU, Kenya \\ *Corresponding Author: okkoech@uonbi.ac.ke
}

Copyright $(0) 2014$ Horizon Research Publishing All rights reserved.

\begin{abstract}
Seed quality and effects of storage on viability of six range grass species grown at 80, 50 and 30\% Field capacity soil water content and rain fed were evaluated. Freshly harvested seeds at week 2 from date of harvest (DOH) had the lowest percent germination. Enteropogon macrostachyus showed low GP $(<10 \%)$ after 2 weeks storage but at the late incubation periods of $11^{\text {th }}$ to $14^{\text {th }}$ day. Sorghum sudanense showed higher GP (> 50\%) after storage period of 2 weeks from $\mathrm{DOH}$ and GP $>75 \%$ from the three soil water content after storage period of 36 weeks. The results also showed after storage periods of 12 weeks, all the grass species start to germination after day 3 of incubation, unlike in the freshly harvested -2 weeks storage period where germination starts after 7 days of incubation except for Sorghum sudanense. Germination index (GI) increased with storage periods for all the grass species with Sorghum sudanense having the highest GI of over 20 after 12 weeks of storage. Storage period of 12 weeks increases seed viability of the six grasses and Sorghum sudanense has higher seed viability even when freshly harvested compared to the other species.
\end{abstract}

Keywords Germination Index, Germination Percentage, Field Capacity, Grass Reseeding, Range Grasses, Grass Seed Quality, Grass Seed Storage, Kenya

\section{Introduction}

Livestock production plays a crucial role in Kenya drylands, with $70 \%$ of livestock and $30 \%$ of human population, and over $80 \%$ of wildlife being supported by these ecosystems [9]. These areas have been under serious degradation, with increasing loss of native grass species that supported livestock population under pastoralism. Rangeland rehabilitation has been reported to be very effective in restoring these degraded areas [30], and this can be achieved through soil conservation by increasing vegetation cover to avoid runoff that depletes the fertile top soil [25]. Ecological restoration through reseeding has also been used to assist degraded areas recover for increased productivity [36] which has been achieved through grass reseeding of degraded Kenyan rangelands with native species [18,22,23]. However, the challenge has always been the source of quality grass seeds adapted to the local condition of the vast rangelands to be rehabilitated [22]. The choice of perennial native grasses has always been promoted due to their ability to improve soil moisture retention and increased infiltration besides protecting the top soil from erosion [20,26,32].

Research efforts are needed on grass seeds multiplication for rehabilitation of denuded areas and pasture establishment for improved pastoral livestock production [3,24,28]. Vegetation and mainly grasses forms an important ecosystem link in terms of energy flow within rangeland ecosystems $[2,14]$. The pastoralist main source of livelihood is livestock keeping and heavily relies on range grasses for products and income from their herds $[21,37]$. The scenario of reduced pastures, increased degradation and loss of native species has worsened their adaptive mechanism threatening their sole livelihood option [31]. For the attainment of millennium development goals (MDGS) and Kenya's vision 2030, of ensuring all citizens have access to adequate and quality food for better lives, a collective action in research and dissemination of information regarding grass seeds and reseeding in the ASALs is vital for the sustainable management of rangeland resources.

Rangeland reseeding study by Mganga [18] reported farmer's efforts to rehabilitated denuded southern rangelands to be affected by poor quality seeds and many efforts by farmers do not show sustainability of their interventions. This is related to use of dormant seeds, too old or too fresh to have reasonable germination rates. For the benefits of reseeding and pasture establishment to be realized, knowledge on seed production and handling under highly variable environment is required [19,24]. 
This study aimed at evaluating the quality of range grass seed produced under irrigation at different soil water content, specifically investigating on seed quality and quality changes with storage periods. Many studies done on range grasses have focused on evaluating the impacts of agronomic management on fodder yields with little attention to water management techniques and their effects on seed production $[1,10]$ contributes to information on irrigation effects on range grass quality which is useful in large scale seed multiplication aiding in increasing farmers access to quality seeds. This is further supported by the present increased demand for rangeland rehabilitation and the increasing demand for livestock forage making grass seed production a priority in the Kenyan rangelands $[8,22]$.

\subsection{Study Area}

This study was conducted from $15^{\text {th }}$ September 2012 to $30^{\text {th }}$ January 2013 in Bura Irrigation Scheme located in Tana River County, Kenya, within coordinates $1^{\circ} 30^{\prime} \mathrm{S}$, $40^{\circ} 0^{\prime} \mathrm{E}, 1.5^{\circ} \mathrm{S} 40^{\circ} \mathrm{E}$ (figure 1). Climate of County is generally hot and dry with daily temperatures ranging between $20^{\circ} \mathrm{C}$ and $38^{\circ} \mathrm{C}$. Rainfall pattern is bimodal with long rains occurring in April-June and short rains in November-December. Long-term average rainfall ranges between $220 \mathrm{~mm}$ and $500 \mathrm{~mm}$ and erratic in distribution. Temperatures are highest between February and April and September to October. About $72 \%$ of the people in the county live below the absolute poverty [9].

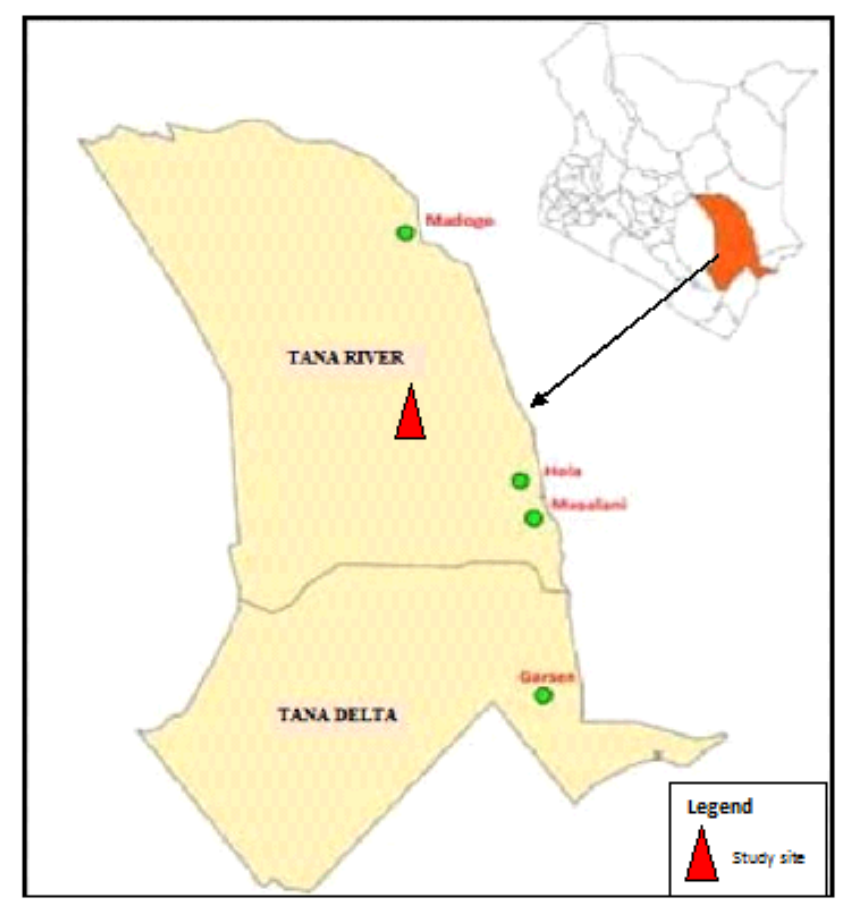

Figure 1. Study area and site

Tana River County is divided into three livelihood zones, namely, pastoral, agro-pastoral (mixed farming) and marginal mixed farming. The soil types in the County are Vertisols and vertic Fluvisols. Dominant vegetation is thorny thickets of acacia trees and shrubs along the riverine areas with shrubs and grasses dominating most parts of the grazing areas. Extensive pastoral system is the main economic activity in the County.

\section{Materials and Methods}

\subsection{Land Preparation, Experimental Design and Agronomic Procedures}

Land that had not been cultivated in previous two seasons was identified within the research site, cleared off bushes, ploughed and harrowed to a fine tilth. The ploughed area was then demarcated into 4 main plots of $39 \mathrm{~m} \mathrm{X} 11 \mathrm{~m}$ which was then randomly allocated soil water content treatments of $80,50,30 \%$ Field capacity (FC) and rainfed conditions. The plots were 5 metres apart to minimize lateral seepage interference. Each main plot was thereafter sub-divided into 30 sub-plots measuring $3 \mathrm{~m} \times 3 \mathrm{~m}$ with $1 \mathrm{~m}$ boundary.

The experimental design was $6 \times 4$ factorial experiments in a completely randomised design comprising two factors, grass species and soil water content at 6 and 4 levels, respectively. The 4 plots demarcated were each randomly assigned to a watering schedule as first treatment. The second treatment level- grass species- was assigned at random to the 30 sub-plots within the 4 main plots. The grass species evaluated were Chloris roxiburghiana- (CR), Eragrostis superba - (ES) Enteropogon macrostachyus (EM), Cenchrus ciliaris - (CC), Chloris gayana - (CG) Sorghum sudanense (Sudan grass- SB).

The watering schedule consisted of different soil moisture recharge levels based on the conventional Field Capacity (FC) determination procedures. Thus, treatment one (T1) was $80 \% \mathrm{FC}$, treatment two (T2) was $50 \% \mathrm{FC}$, treatment three (T3) was 30\% FC and treatment four (T4) was the control (rain fed). For each treatment, soil moisture was maintained at the prescribed level through irrigation. In order to know when to recharge the plots, soil moisture content was monitored by means of the Delmhorst Soil Moisture Meter Gypsum Blocks (GBs) installed within each sub-plots.

The grass seeds used were sourced from Kenya Agricultural Research Institute (KARI), Kiboko Station. The seeds were tested for germination percentage using the standard seed test by germination method as described by [13]. The germination rates obtained were used to determine the mixing and sowing rates of the species. Sowing was done manually on the finely prepared seedbeds on the subplots at the predetermined sowing rates. Phosphate fertilizer was applied to all the treatments at the recommended rate of $200 \mathrm{~kg} / \mathrm{ha}$ to enhance establishment.

\subsection{Seed Harvest}

Seed yields were determined by hand harvesting of 
mature and ripened seeds within a $(1 \mathrm{mX} 1 \mathrm{~m})$ systematically placed quadrat replicated three times on the six species sub-plots. This was done for each species at the time of full maturity and ripened seeds. The seeds were stored in labelled brown paper bags, then oven dried at $60^{\circ} \mathrm{C}$ for 48 hours, before quality determination with storage periods.

\subsection{Seed Quality Determination}

Seed quality was estimated by germination percent (GP) and germination index (GI). The seeds harvested from the six grass species grown under the three soil water content levels and rainfed were tested for quality as an indication of dormancy over a period of 36 months. The seeds were stored in brown papers at room temperature $\left(28^{\circ} \mathrm{C}\right)$. Storage period as a factor of breaking seed dormancy has been reported by many studies $[7,18,33]$. Procedures followed in determining GP and GI are described below.

\subsection{Germination Percent with Storage Periods}

Germination percentage (GP) of the seeds after storage periods of weeks 2, 12, 24 and 36, was determined following procedures provided by International Seed Testing Association [12] of petri-dish germination method described by [34] and [17]. ISTA [13] recommends use of seed germination percentage to test the quality of seeds as a standard measure of seed quality. The methodology involved random selection of 100 seeds of each of the six grass species under evaluation, replicated 4 times. The seeds were then put on Whitman filter paper in a petridish under room temperature $\left(28^{\circ} \mathrm{C}\right)$ at the University of Nairobi, range livestock nutrition laboratory. The seeds were incubated for 14 days and monitored for germination. All the germinated seeds were expressed as a percentage of the total number of seeds incubated during the period. This procedure was done to all the grass species at the end of 2 , 12,24 and 36 weeks storage periods from the date of harvest $(\mathrm{DOH})$. The percent germination was calculated using the equation 1 below:

$\%$ Germination $=\frac{\text { Total number of seeds germinated } \mathrm{X} 100}{\text { Seeds per petri-dish } \times \text { Replicates }} \ldots$

\subsection{Germination Index}

Germination index as an indication of speed of germination of the six grass seeds was determined by picking random samples of 100 seeds for each grass species obtained from the different soil water content harvest, which were then put on a wet Whatman filter paper in standard laboratory petri-dishes. Each petri-dish had specific grass species with 25 grass seeds (caryopsis) replicated four (4) times. The petri-dish was then incubated in open air room temperature for 14 days at the University of Nairobi, range livestock nutrition laboratory. The filter paper was moistened with a few drops of water when it appeared dry. The grass seeds that germinated everyday were counted and removed from the petri-dishes. This was done after a clear identifiable radical was observed [27]. The number of seedlings emerging daily was counted from day of incubation to $14^{\text {th }}$ day. Thereafter, Germination index (G.I.) was computed by use of the following formula (Equation 2).

$$
G I=n / d
$$

Where, $\mathrm{n}=$ number of seedlings emerging on day' $\mathrm{d}^{\prime}$ and $\mathrm{d}=$ day after planting.

Grass seeds that showed greater germination index were considered to be more vigorous and of better quality [12].

\section{Results}

\section{1. Germination Percent (GP)}

Tables 1, 2, 3 and 4 presents the daily percent seed germination of the six grass species obtained from 80, 50, $30 \%$ FC soil water content and rainfed conditions, respectively, after storage periods of 2, 12, 24 and 36 weeks from DOH. There was significant difference $(\mathrm{p} \leq 0.05)$ in seed germination percent for different grass species at week 12 , with others showing higher and others low percent seed germination across the 80,50 and $30 \% \mathrm{FC}$ soil water content levels. Generally, an increase percent seed germination was observed with storage periods, where freshly harvested seeds at week 2 from DOH having lowest percent germination and at week 36 storage, all species showing above 50\% germination percent. There was no significant difference in GP for specific species across the three soil water content and rain fed treatments. Chloris roxburghina, Eragrostis superba and Cenchrus ciliaris showed no germination after 2 weeks of storage from DOH at the three soil water content and rain fed treatment. Enteropogon macrostachyus showed low germination percent $(<10 \%)$ after 2 weeks storage but at the late incubation periods (from day 11 to 14 ).

Sorghum sudanense showed higher germination percent (over 50\%) after storage period of 2 weeks from $\mathrm{DOH}$ for the three soil water content, however, it had $<50 \%$ germination at the same storage period under rain fed condition. Sorghum sudanense still showed higher percent germination $(>75 \%)$ for watered conditions after storage period of 36 weeks from $\mathrm{DOH}$, while under rain fed, just like with the two weeks storage period, still had lower germination percent compared to watered treatments, however was not significant different. The results showed after storage periods of 12 weeks, all the species start to germination after day 3 of incubation. 
Table 1. Daily \% seed germination of six range grass species after storage period of 2, 12, 24 and 36 weeks from DOH, produced at $80 \%$ FC soil water content

\begin{tabular}{|c|c|c|c|c|c|c|c|c|c|c|c|c|c|c|}
\hline Days & 1 & 2 & 3 & 4 & 5 & 6 & 7 & 8 & 9 & 10 & 11 & 12 & 13 & 14 \\
\hline \multicolumn{15}{|c|}{ Week 2} \\
\hline C R & 0 & 0 & 0 & 0 & 0 & 0 & 0 & 0 & 0 & 0 & 0 & 0 & 0 & 0 \\
\hline E S & 0 & 0 & 0 & 0 & 0 & 0 & 0 & 0 & 0 & 0 & 0 & 0 & 0 & 0 \\
\hline EM & 0 & 0 & 0 & 0 & 0 & 0 & 0 & 0 & 0 & 0 & 6.1 & $6.4^{\mathrm{a}}$ & $8.5^{\mathrm{a}}$ & $8.8^{\mathrm{a}}$ \\
\hline $\mathrm{CC}$ & 0 & 0 & 0 & 0 & 0 & 0 & 0 & 0 & 0 & 0 & 0 & 0 & 0 & 0 \\
\hline $\mathrm{CG}$ & 0 & 0 & 0 & 0 & 0 & 0 & 0 & $8.2^{\mathrm{a}}$ & $10.4^{\mathrm{a}}$ & $10.5^{\mathrm{a}}$ & $10.2^{\mathrm{a}}$ & $11.4^{\mathrm{a}}$ & $11.8^{\mathrm{a}}$ & $14.5^{\mathrm{a}}$ \\
\hline SB & 0 & 0 & $5.2^{\mathrm{a}}$ & $6.3^{\mathrm{a}}$ & $11.4^{\mathrm{a}}$ & $12.0^{\mathrm{a}}$ & $12.5^{\mathrm{a}}$ & $16.1^{\mathrm{a}}$ & $17.4^{\mathrm{a}}$ & $31.5^{\mathrm{a}}$ & $39.8^{\mathrm{b}}$ & $45.5^{\mathrm{b}}$ & $60.5^{\mathrm{c}}$ & $60.3^{c}$ \\
\hline \multicolumn{15}{|c|}{ Week 12} \\
\hline C R & 0 & 0 & 0 & $8.3^{\mathrm{a}}$ & $8.0^{\mathrm{a}}$ & $10.2^{\mathrm{a}}$ & $11.5^{\mathrm{a}}$ & $11.2^{\mathrm{a}}$ & $12.4^{\mathrm{a}}$ & $12.6^{\mathrm{a}}$ & $12.5^{\mathrm{a}}$ & $14.2^{\mathrm{a}}$ & $16 .^{\mathrm{a}}$ & $21.2^{\mathrm{b}}$ \\
\hline E S & 0 & 0 & $3.1^{\mathrm{a}}$ & $6.5^{\mathrm{a}}$ & $7.1^{\mathrm{a}}$ & $7.0^{\mathrm{a}}$ & $8.5^{\mathrm{a}}$ & $10.2^{\mathrm{a}}$ & $13.1^{\mathrm{a}}$ & $13.0^{\mathrm{a}}$ & $15.2^{\mathrm{a}}$ & $14.9^{\mathrm{a}}$ & $16.7^{\mathrm{a}}$ & $19.8^{\mathrm{a}}$ \\
\hline EM & 0 & 0 & $11.2^{\mathrm{a}}$ & $15.4^{\mathrm{a}}$ & $15.5^{\mathrm{a}}$ & $15.9^{\mathrm{a}}$ & $16.7^{\mathrm{a}}$ & $18.6^{\mathrm{a}}$ & $22.1^{\mathrm{b}}$ & $22.0^{\mathrm{a}}$ & $25.0^{\mathrm{a}}$ & $24.8^{\mathrm{a}}$ & $26.5^{\mathrm{b}}$ & $26.6^{\mathrm{b}}$ \\
\hline $\mathrm{CC}$ & 0 & 0 & $4.5^{\mathrm{a}}$ & $5.5^{\mathrm{a}}$ & $5.3^{\mathrm{a}}$ & $7.8^{\mathrm{a}}$ & $7.0^{\mathrm{a}}$ & $7.2^{\mathrm{a}}$ & $9.7^{\mathrm{a}}$ & $9.3^{\mathrm{a}}$ & $8.7^{\mathrm{a}}$ & $8.5^{\mathrm{a}}$ & $10.2^{\mathrm{a}}$ & $13.1^{\mathrm{a}}$ \\
\hline $\mathrm{CG}$ & 0 & $1.1^{\mathrm{a}}$ & $13.0^{\mathrm{a}}$ & $15.7^{\mathrm{a}}$ & $16.1^{\mathrm{a}}$ & $19.3^{\mathrm{a}}$ & $20.4^{\mathrm{a}}$ & $37.0^{\mathrm{b}}$ & $38.5^{\mathrm{b}}$ & $44.5^{\mathrm{b}}$ & $49.7^{c}$ & $51.9^{\mathrm{a}}$ & $54.5^{\mathrm{c}}$ & $58.7^{\mathrm{c}}$ \\
\hline SB & 0 & 0 & $18.2^{\mathrm{a}}$ & $26.3^{\mathrm{b}}$ & $27.4^{\mathrm{b}}$ & $32.0^{\mathrm{b}}$ & $32.5^{\mathrm{b}}$ & $46.1^{\mathrm{c}}$ & $57.4^{\mathrm{c}}$ & $61.5^{\mathrm{c}}$ & $69.8^{\mathrm{c}}$ & $65.5^{\mathrm{c}}$ & $70.5^{\mathrm{d}}$ & $70.3^{d}$ \\
\hline \multicolumn{15}{|c|}{ Week 24} \\
\hline C R & 0 & 0 & $10.5^{\mathrm{a}}$ & $19.5^{\mathrm{a}}$ & $29.1^{\mathrm{b}}$ & $29.4^{\mathrm{b}}$ & $29.5^{\mathrm{b}}$ & $33.5^{\mathrm{b}}$ & $37.4^{\mathrm{b}}$ & $38.6^{\mathrm{b}}$ & $37.5^{\mathrm{b}}$ & $51.2^{\mathrm{c}}$ & 56.1 & $54.7^{\mathrm{c}}$ \\
\hline E S & 0 & 0 & $26.0^{\mathrm{b}}$ & $28.5^{\mathrm{a}}$ & $47.1^{\mathrm{c}}$ & $47.0^{\mathrm{c}}$ & $48.5^{\mathrm{c}}$ & $51.2^{\mathrm{c}}$ & $53.4^{\mathrm{c}}$ & $53.0^{\mathrm{c}}$ & $55.2^{\mathrm{c}}$ & $54.9^{\mathrm{c}}$ & $56.7^{\mathrm{c}}$ & $59.8^{\mathrm{c}}$ \\
\hline EM & 0 & 0 & $31.4^{\mathrm{b}}$ & $35.5^{\mathrm{b}}$ & $35.8^{\mathrm{b}}$ & $35.9^{\mathrm{b}}$ & $36.8^{\mathrm{b}}$ & $38.2^{\mathrm{b}}$ & $45.1^{\mathrm{b}}$ & $52.0^{c}$ & $55.0^{\mathrm{c}}$ & $59.4^{\mathrm{c}}$ & $61.3^{\mathrm{c}}$ & $66.7^{\mathrm{c}}$ \\
\hline $\mathrm{CC}$ & 0 & 0 & $30.2^{\mathrm{b}}$ & $35.5^{\mathrm{b}}$ & $35.2^{\mathrm{b}}$ & $37.9^{\mathrm{b}}$ & $37.9^{\mathrm{b}}$ & $42.2^{\mathrm{b}}$ & $49.1^{\mathrm{b}}$ & $49.7^{\mathrm{c}}$ & $49.9^{\mathrm{c}}$ & $51.5^{\mathrm{c}}$ & $55.8^{\mathrm{c}}$ & $57.9^{\mathrm{c}}$ \\
\hline CG & 0 & 0 & $18.0^{\mathrm{a}}$ & $25.7^{\mathrm{b}}$ & $26.7^{\mathrm{b}}$ & $39.3^{\mathrm{b}}$ & $38.9^{\mathrm{b}}$ & $37.0^{\mathrm{b}}$ & $42.4^{\mathrm{b}}$ & $47.3^{c}$ & $46.8^{\mathrm{c}}$ & $52.4^{\mathrm{c}}$ & $51.9^{\mathrm{c}}$ & $57.4^{\mathrm{c}}$ \\
\hline SB & 0 & 0 & $55.8^{\mathrm{c}}$ & $59.5^{\mathrm{c}}$ & $57.5^{\mathrm{c}}$ & $59.0^{\mathrm{c}}$ & $65.5^{\mathrm{c}}$ & $65.9^{\mathrm{c}}$ & $69.1^{\mathrm{c}}$ & $69.5^{\mathrm{c}}$ & $69.6^{\mathrm{c}}$ & $71.5^{\mathrm{d}}$ & $75.5^{\mathrm{d}}$ & $73.3^{d}$ \\
\hline \multicolumn{15}{|c|}{ Week 36} \\
\hline C R & 0 & 0 & $29.7^{\mathrm{b}}$ & $30.4^{\mathrm{b}}$ & $32.1^{\mathrm{b}}$ & $32.4^{\mathrm{b}}$ & $36.8^{\mathrm{b}}$ & $37.9^{\mathrm{b}}$ & $39.8^{\mathrm{b}}$ & $42.5^{\mathrm{b}}$ & $46.2^{\mathrm{b}}$ & $47.8^{\mathrm{c}}$ & $52.8^{\mathrm{c}}$ & $57.2^{\mathrm{c}}$ \\
\hline E S & 0 & 0 & $27.1^{\mathrm{b}}$ & $27.5^{\mathrm{b}}$ & $57.1^{\mathrm{c}}$ & $57.8^{\mathrm{c}}$ & $58.2^{\mathrm{c}}$ & $58.5^{\mathrm{c}}$ & $59.4^{\mathrm{c}}$ & $61.0^{\mathrm{c}}$ & $62.3^{\mathrm{c}}$ & $64.9^{\mathrm{c}}$ & $65.1^{\mathrm{c}}$ & $65.3^{\mathrm{c}}$ \\
\hline EM & 0 & 0 & $33.5^{\mathrm{b}}$ & $35.0^{\mathrm{b}}$ & $36.2^{\mathrm{b}}$ & $36.9^{c}$ & $43.8^{\mathrm{b}}$ & $45.8^{\mathrm{b}}$ & $55.3^{\mathrm{c}}$ & $62.0^{\mathrm{c}}$ & $65.8^{\mathrm{c}}$ & $69.6^{\mathrm{c}}$ & $66.3^{\mathrm{c}}$ & $69.8^{\mathrm{c}}$ \\
\hline $\mathrm{CC}$ & 0 & 0 & $40.0^{\mathrm{b}}$ & $41.8^{\mathrm{b}}$ & $42.5^{\mathrm{b}}$ & $45.2^{\mathrm{c}}$ & $45.2^{\mathrm{b}}$ & $45.1^{\mathrm{b}}$ & $45.8^{\mathrm{b}}$ & $48.8^{c}$ & $50.0^{\mathrm{c}}$ & $53.6^{\mathrm{c}}$ & $57.5^{\mathrm{c}}$ & $61.4^{\mathrm{c}}$ \\
\hline $\mathrm{CG}$ & 0 & 0 & $21.1^{\mathrm{a}}$ & $23.8^{\mathrm{a}}$ & $36.7^{\mathrm{b}}$ & $39.8^{\mathrm{b}}$ & $42.1^{\mathrm{b}}$ & $43.0^{\mathrm{b}}$ & $45.3^{\mathrm{b}}$ & $48.6^{\mathrm{c}}$ & $51.3^{\mathrm{c}}$ & $58.6^{\mathrm{c}}$ & $61.4^{\mathrm{c}}$ & $67.9^{c}$ \\
\hline SB & 0 & 0 & $53.8^{\mathrm{c}}$ & $58.5^{\mathrm{c}}$ & $59.0^{\mathrm{c}}$ & $59.2^{\mathrm{c}}$ & $66.4^{\mathrm{c}}$ & $68.9^{\mathrm{c}}$ & $69.1^{\mathrm{c}}$ & $69.4^{\mathrm{c}}$ & $69.7^{\mathrm{c}}$ & $71.5^{\mathrm{c}}$ & $74.6^{\mathrm{d}}$ & $75.2^{\mathrm{d}}$ \\
\hline
\end{tabular}

Means within the same columns with different superscripts are significantly different at $\mathrm{p}<0.05$.

Key: $C R=$ Chloris roxburghiana, $E S=$ Eragrostis superba, $E M=$ Enteropogon macrostachyus, $C C=$ Cenchrus ciliaris, $C G=$ Chloris gayana, $S B=$ 
Table 2. Daily \% seed germination of six range grass species after storage period of 2, 12, 24 and 36 weeks from DOH, produced at $50 \%$ FC soil water content

\begin{tabular}{|c|c|c|c|c|c|c|c|c|c|c|c|c|c|c|}
\hline Days & 1 & 2 & 3 & 4 & 5 & 6 & 7 & 8 & 9 & 10 & 11 & 12 & 13 & 14 \\
\hline \multicolumn{15}{|c|}{ Week 2} \\
\hline C R & 0 & 0 & 0 & 0 & 0 & 0 & 0 & 0 & 0 & 0 & 0 & 0 & 0 & 0 \\
\hline E S & 0 & 0 & 0 & 0 & 0 & 0 & 0 & 0 & 0 & 0 & 0 & 0 & 0 & 0 \\
\hline EM & 0 & 0 & 0 & 0 & 0 & 0 & 0 & 0 & $2.3^{\mathrm{a}}$ & $4.5^{\mathrm{a}}$ & $5.2^{\mathrm{a}}$ & $6.1^{\mathrm{a}}$ & $6.4^{\mathrm{a}}$ & $9.2^{\mathrm{a}}$ \\
\hline $\mathrm{CC}$ & 0 & 0 & 0 & 0 & 0 & 0 & 0 & 0 & 0 & 0 & 0 & 0 & 0 & 0 \\
\hline CG & 0 & 0 & 0 & 0 & 0 & 0 & 0 & $7.0^{\mathrm{a}}$ & $8.0^{\mathrm{a}}$ & $9.1^{\mathrm{a}}$ & $9.6^{\mathrm{a}}$ & $10.2^{\mathrm{a}}$ & $10.8^{\mathrm{a}}$ & $12.1^{\mathrm{a}}$ \\
\hline SB & 0 & 0 & $2.5^{\mathrm{a}}$ & $4.1^{\mathrm{a}}$ & $8.6^{\mathrm{a}}$ & $12.3^{\mathrm{a}}$ & $11.5^{\mathrm{a}}$ & $15.1^{\mathrm{a}}$ & $16.9^{\mathrm{a}}$ & $28.5^{\mathrm{b}}$ & $29.8^{\mathrm{b}}$ & $35.5^{\mathrm{b}}$ & $44.5^{\mathrm{b}}$ & $59.1^{\circ}$ \\
\hline \multicolumn{15}{|c|}{ Week 12} \\
\hline C R & 0 & 0 & 0 & $7.8^{\mathrm{a}}$ & $8.1^{\mathrm{a}}$ & $9.3^{\mathrm{a}}$ & $10.5^{\mathrm{a}}$ & $10.8^{\mathrm{a}}$ & $11.3^{\mathrm{a}}$ & $12.0^{\mathrm{a}}$ & $13.4^{\mathrm{a}}$ & $14.0^{\mathrm{a}}$ & $15.8^{\mathrm{a}}$ & $19.5^{\mathrm{a}}$ \\
\hline E S & 0 & 0 & $4.0^{\mathrm{a}}$ & $5.2^{\mathrm{a}}$ & $6.3^{\mathrm{a}}$ & $6.6^{\mathrm{a}}$ & $9.0^{\mathrm{a}}$ & $9.2^{\mathrm{a}}$ & $10.4^{\mathrm{a}}$ & $12.3^{\mathrm{a}}$ & $14.1^{\mathrm{a}}$ & $15.9^{\mathrm{a}}$ & $17.4^{\mathrm{a}}$ & $22.1^{\mathrm{a}}$ \\
\hline EM & 0 & 0 & $10.2^{\mathrm{a}}$ & $13.4^{\mathrm{a}}$ & $14.3^{\mathrm{a}}$ & $16.0^{\mathrm{a}}$ & $16.6^{\mathrm{a}}$ & $19.2^{\mathrm{a}}$ & $23.1^{\mathrm{b}}$ & $24.5^{\mathrm{a}}$ & $25.7^{\mathrm{b}}$ & $28.7^{\mathrm{b}}$ & $29.5^{\mathrm{b}}$ & $29.9^{\mathrm{a}}$ \\
\hline $\mathrm{CC}$ & 0 & 0 & $3.0^{\mathrm{a}}$ & $4.5^{\mathrm{a}}$ & $6.0^{\mathrm{a}}$ & $7.3^{\mathrm{a}}$ & $7.6^{\mathrm{a}}$ & $8.0^{\mathrm{a}}$ & $9.5^{\mathrm{a}}$ & $10.1^{\mathrm{a}}$ & $11.0^{\mathrm{a}}$ & $12.5^{\mathrm{a}}$ & $12.9^{\mathrm{a}}$ & $13.6^{\mathrm{a}}$ \\
\hline CG & 0 & 0 & $8.0^{\mathrm{a}}$ & $8.7^{\mathrm{a}}$ & $15.1^{\mathrm{a}}$ & $17.5^{\mathrm{a}}$ & $21.4^{\mathrm{b}}$ & $27.0^{\mathrm{b}}$ & $28.6^{\mathrm{b}}$ & $34.9^{\mathrm{b}}$ & $44.7^{\mathrm{b}}$ & $48.6^{\mathrm{b}}$ & $53.0^{\mathrm{c}}$ & $54.0^{\circ}$ \\
\hline SB & 0 & 0 & $21.5^{\mathrm{b}}$ & $27.8^{\mathrm{b}}$ & $29.5^{\mathrm{b}}$ & $33.0^{\mathrm{b}}$ & $35.6^{\mathrm{b}}$ & $44.1^{\mathrm{b}}$ & $47.3^{\mathrm{b}}$ & $51.5^{\mathrm{c}}$ & $59.6^{\mathrm{c}}$ & $63.0^{\mathrm{c}}$ & $65.2^{\mathrm{c}}$ & $68.0^{\mathrm{b}}$ \\
\hline \multicolumn{15}{|c|}{ Week 24} \\
\hline $\mathrm{C} \mathrm{R}$ & 0 & 0 & $8.6^{\mathrm{a}}$ & $15.3^{\mathrm{b}}$ & $25.2^{\mathrm{b}}$ & $29.0^{\mathrm{b}}$ & $30.0^{\mathrm{b}}$ & $36.2^{\mathrm{b}}$ & $38.0^{\mathrm{b}}$ & $42.4^{\mathrm{b}}$ & $47.0^{\mathrm{b}}$ & $50.1^{\mathrm{c}}$ & $50.5^{\mathrm{c}}$ & $52.0^{\circ}$ \\
\hline E S & 0 & 0 & $21.0^{\mathrm{b}}$ & $22.8^{\mathrm{b}}$ & $27.5^{\mathrm{b}}$ & $37.0^{\mathrm{b}}$ & $42.5^{\mathrm{b}}$ & $50.0^{\mathrm{c}}$ & $52.3^{\mathrm{c}}$ & $53.5^{\mathrm{c}}$ & $54.0^{\mathrm{c}}$ & $57.0^{\mathrm{c}}$ & $59.9^{\mathrm{c}}$ & $61.9^{\circ}$ \\
\hline EM & 0 & 0 & $28.0^{\mathrm{b}}$ & $30.7^{\mathrm{b}}$ & $36.0^{\mathrm{b}}$ & $39.0^{\mathrm{b}}$ & $41.5^{\mathrm{b}}$ & $42.3^{\mathrm{b}}$ & $43.0^{\mathrm{b}}$ & $50.2^{\mathrm{c}}$ & $53.1^{\mathrm{c}}$ & $55.5^{\mathrm{c}}$ & $59.8^{\mathrm{c}}$ & $68.0^{\circ}$ \\
\hline $\mathrm{CC}$ & 0 & 0 & $28.0^{\mathrm{b}}$ & $33.0^{\mathrm{b}}$ & $36.1^{\mathrm{b}}$ & $38.2^{\mathrm{b}}$ & $40.4^{\mathrm{b}}$ & $44.0^{\mathrm{b}}$ & $45.3^{\mathrm{b}}$ & $48.7^{\mathrm{b}}$ & $48.3^{\mathrm{b}}$ & $53.7^{\mathrm{c}}$ & $55.0^{\mathrm{c}}$ & $55.3^{\mathrm{c}}$ \\
\hline CG & 0 & 0 & $9.0^{\mathrm{a}}$ & $24.3^{\mathrm{b}}$ & $28.6^{\mathrm{b}}$ & $37.0^{\mathrm{b}}$ & $41.0^{\mathrm{b}}$ & $43.1^{\mathrm{b}}$ & $45.0^{\mathrm{b}}$ & $45.5^{\mathrm{b}}$ & $48.2^{\mathrm{b}}$ & $50.0^{\mathrm{c}}$ & $55.1^{\mathrm{c}}$ & $59.2^{\circ}$ \\
\hline SB & 0 & 0 & $52.1^{\mathrm{c}}$ & $55.1^{\mathrm{c}}$ & $58.0^{\mathrm{c}}$ & $61.0^{\mathrm{c}}$ & $63.1^{\mathrm{c}}$ & $65.2^{\mathrm{b}}$ & $67.0^{\mathrm{c}}$ & $70.5^{\mathrm{c}}$ & $71.0^{\mathrm{d}}$ & $72.0^{\mathrm{d}}$ & $72.9^{\mathrm{d}}$ & $76.5^{\mathrm{d}}$ \\
\hline \multicolumn{15}{|c|}{ Week 36} \\
\hline $\mathrm{CR}$ & 0 & 0 & $25.0^{\mathrm{b}}$ & $29.0^{\mathrm{b}}$ & $30.1^{\mathrm{b}}$ & $32.1^{\mathrm{b}}$ & $35.2^{\mathrm{b}}$ & $35.6^{\mathrm{b}}$ & $40.2^{\mathrm{b}}$ & $45.1^{\mathrm{b}}$ & $48.0^{\mathrm{b}}$ & $50.5^{\mathrm{c}}$ & $53.5^{\mathrm{c}}$ & $58.0^{\circ}$ \\
\hline E S & 0 & 0 & $20.0^{\mathrm{b}}$ & $25.2^{\mathrm{b}}$ & $45.3^{\mathrm{b}}$ & $55.1^{\mathrm{c}}$ & $57.8^{\mathrm{c}}$ & $59.0^{\mathrm{b}}$ & $60.2^{\mathrm{c}}$ & $63.1^{\mathrm{c}}$ & $64.1^{\mathrm{c}}$ & $65.0^{\mathrm{c}}$ & $67.2^{\mathrm{c}}$ & $68.0^{\circ}$ \\
\hline EM & 0 & 0 & $27.0^{\mathrm{b}}$ & $30.2^{\mathrm{b}}$ & $35.0^{\mathrm{b}}$ & $38.8^{\mathrm{b}}$ & $40.2^{\mathrm{b}}$ & $44.0^{\mathrm{b}}$ & $49.2^{\mathrm{b}}$ & $58.6^{\mathrm{c}}$ & $63.0^{\mathrm{c}}$ & $65.2^{\mathrm{c}}$ & $69.1^{\mathrm{c}}$ & $70.1^{\mathrm{d}}$ \\
\hline $\mathrm{CC}$ & 0 & 0 & $25.0^{\mathrm{b}}$ & $31.2^{\mathrm{b}}$ & $40.5^{\mathrm{b}}$ & $43.5^{\mathrm{b}}$ & $46.0^{\mathrm{b}}$ & $46.9^{b}$ & $50.3^{\mathrm{c}}$ & $52.2^{\mathrm{c}}$ & $54.0^{\mathrm{b}}$ & $61.0^{c}$ & $62.1^{\mathrm{c}}$ & $65.1^{\circ}$ \\
\hline CG & 0 & 0 & $28.1^{\mathrm{b}}$ & $33.2^{\mathrm{b}}$ & $35.3^{\mathrm{b}}$ & $40.8^{\mathrm{b}}$ & $43.0^{\mathrm{b}}$ & $45.2^{\mathrm{b}}$ & $47.1^{\mathrm{b}}$ & $50.5^{\mathrm{c}}$ & $55.2^{\mathrm{b}}$ & $59.0^{\mathrm{c}}$ & $64.6^{\mathrm{c}}$ & $69.0^{\circ}$ \\
\hline SB & 0 & 0 & $55.0^{\mathrm{c}}$ & $58.2^{\mathrm{c}}$ & $60.1^{\mathrm{c}}$ & $62.0^{\mathrm{c}}$ & $64.2^{\mathrm{c}}$ & $66.6^{\mathrm{c}}$ & $67.9^{\mathrm{d}}$ & $72.1^{\mathrm{d}}$ & $74.1^{\mathrm{d}}$ & $73.0^{\mathrm{d}}$ & $74.2^{\mathrm{d}}$ & $75.0^{\mathrm{d}}$ \\
\hline
\end{tabular}

Means within the same columns with different superscripts are significantly different at $\mathrm{p}<0.05$.

Key: $C R=$ Chloris roxburghiana, $E S=$ Eragrostis superba, $E M=$ Enteropogon macrostachyus, $C C=$ Cenchrus ciliaris, $C G=$ Chloris gayana, $S B=S o r g h u m$ sudanense 
Table 3. Daily $\%$ seed germination of six range grass species after storage period of 2, 12,24 and 36 weeks from DOH, produced at $30 \%$ FC soil water content

\begin{tabular}{|c|c|c|c|c|c|c|c|c|c|c|c|c|c|c|}
\hline Days & 1 & 2 & 3 & 4 & 5 & 6 & 7 & 8 & 9 & 10 & 11 & 12 & 13 & 14 \\
\hline \multicolumn{15}{|c|}{ Week 2} \\
\hline C R & 0 & 0 & 0 & 0 & 0 & 0 & 0 & 0 & 0 & 0 & 0 & 0 & 0 & 0 \\
\hline E S & 0 & 0 & 0 & 0 & 0 & 0 & 0 & 0 & 0 & 0 & 0 & 0 & 0 & 0 \\
\hline EM & 0 & 0 & 0 & 0 & 0 & 0 & 0 & 0 & $2.0^{\mathrm{a}}$ & $3.5^{\mathrm{a}}$ & $4.9^{\mathrm{a}}$ & $5.8^{\mathrm{a}}$ & $6.0^{\mathrm{a}}$ & $8.5^{\mathrm{a}}$ \\
\hline $\mathrm{CC}$ & 0 & 0 & 0 & 0 & 0 & 0 & 0 & 0 & 0 & 0 & 0 & 0 & 0 & 0 \\
\hline CG & 0 & 0 & 0 & 0 & 0 & 0 & 0 & $4.1^{\mathrm{a}}$ & $7.0^{\mathrm{a}}$ & $8.0^{\mathrm{a}}$ & $9.5^{\mathrm{a}}$ & $11.1^{\mathrm{a}}$ & $11.8^{\mathrm{b}}$ & $12.4^{\mathrm{a}}$ \\
\hline SB & 0 & 0 & $3.0^{\mathrm{a}}$ & $5.1^{\mathrm{a}}$ & $6.5^{\mathrm{a}}$ & $11.3^{\mathrm{a}}$ & $12.6^{\mathrm{b}}$ & $14.3^{\mathrm{a}}$ & $15.3^{\mathrm{a}}$ & $30.1^{\mathrm{a}}$ & $33.8^{\mathrm{b}}$ & $34.2^{\mathrm{b}}$ & $40.5^{\mathrm{b}}$ & $58.0^{\mathrm{c}}$ \\
\hline \multicolumn{15}{|c|}{ Week 12} \\
\hline C R & 0 & 0 & $3.0^{\mathrm{a}}$ & $5.1^{\mathrm{a}}$ & $7.0^{\mathrm{a}}$ & $8.0^{\mathrm{a}}$ & $10.0^{\mathrm{a}}$ & $11.7^{\mathrm{a}}$ & $11.9^{\mathrm{a}}$ & $12.4^{\mathrm{a}}$ & $13.7^{\mathrm{a}}$ & $14.2^{\mathrm{a}}$ & $14.8^{\mathrm{a}}$ & $18.3^{\mathrm{a}}$ \\
\hline E S & 0 & 0 & $5.0^{\mathrm{a}}$ & $5.7^{\mathrm{a}}$ & $6.0^{\mathrm{a}}$ & $7.0^{\mathrm{a}}$ & $9.5^{\mathrm{a}}$ & $10.2^{\mathrm{a}}$ & $11.6^{\mathrm{a}}$ & $12.0^{\mathrm{a}}$ & $13.2^{\mathrm{a}}$ & $14.1^{\mathrm{a}}$ & $15.0^{\mathrm{a}}$ & $19.5^{\mathrm{a}}$ \\
\hline EM & 0 & 0 & $12.0^{\mathrm{a}}$ & $14.1^{\mathrm{a}}$ & $15.5^{\mathrm{a}}$ & $17.2^{\mathrm{a}}$ & $18.6^{\mathrm{a}}$ & $19.4^{\mathrm{a}}$ & $22.5^{\mathrm{a}}$ & $25.0^{\mathrm{b}}$ & $27.1^{\mathrm{c}}$ & $31.5^{\mathrm{b}}$ & $35.5^{\mathrm{b}}$ & $42.8^{\mathrm{b}}$ \\
\hline $\mathrm{CC}$ & 0 & 0 & $4.0^{\mathrm{a}}$ & $6.1^{\mathrm{a}}$ & $8.0^{\mathrm{a}}$ & $10.2^{\mathrm{a}}$ & $11.3^{\mathrm{a}}$ & $12.2^{\mathrm{a}}$ & $13.4^{\mathrm{a}}$ & $14.9^{\mathrm{a}}$ & $15.6^{\mathrm{b}}$ & $18.0^{\mathrm{a}}$ & $22.2^{\mathrm{b}}$ & $29.5^{\mathrm{b}}$ \\
\hline CG & 0 & 0 & $6.0^{\mathrm{a}}$ & $10.3^{\mathrm{a}}$ & $16.0^{\mathrm{a}}$ & $18.8^{\mathrm{a}}$ & $22.1^{\mathrm{b}}$ & $26.5^{\mathrm{b}}$ & $29.3^{\mathrm{b}}$ & $32.4^{\mathrm{b}}$ & $41.8^{\mathrm{b}}$ & $47.3^{\mathrm{b}}$ & $51.2^{\mathrm{c}}$ & $53.4^{\mathrm{c}}$ \\
\hline SB & 0 & 0 & $24.5^{\mathrm{b}}$ & $29.3^{\mathrm{b}}$ & $29.0^{\mathrm{b}}$ & $32.1^{\mathrm{b}}$ & $34.8^{\mathrm{b}}$ & $42.0^{\mathrm{b}}$ & $45.5^{\mathrm{b}}$ & $49.8^{\mathrm{b}}$ & $54.3^{\mathrm{c}}$ & $58.0^{\mathrm{c}}$ & $62.7^{\mathrm{c}}$ & $65.4^{\mathrm{c}}$ \\
\hline \multicolumn{15}{|c|}{ Week 24} \\
\hline C R & 0 & 0 & $3.0^{\mathrm{a}}$ & $9.5^{\mathrm{a}}$ & $15.2^{\mathrm{a}}$ & $13.5^{\mathrm{a}}$ & $25.6^{\mathrm{b}}$ & $33.1^{\mathrm{b}}$ & $35.0^{\mathrm{b}}$ & $41.5^{\mathrm{b}}$ & $45.0^{\mathrm{b}}$ & $47.2^{\mathrm{b}}$ & $51.6^{\mathrm{c}}$ & $54.0^{\mathrm{b}}$ \\
\hline E S & 0 & 0 & $16.3^{\mathrm{a}}$ & $21.9^{\mathrm{b}}$ & $26.0^{\mathrm{b}}$ & $35.0^{\mathrm{b}}$ & $40.1^{\mathrm{b}}$ & $45.5^{\mathrm{b}}$ & $47.2^{\mathrm{b}}$ & $51.0^{\mathrm{c}}$ & $53.2^{\mathrm{c}}$ & $54.0^{\mathrm{c}}$ & $55.4^{\mathrm{c}}$ & $59.6^{\mathrm{b}}$ \\
\hline EM & 0 & 0 & $24.5^{\mathrm{b}}$ & $33.8^{\mathrm{b}}$ & $39.2^{\mathrm{b}}$ & $42.0^{\mathrm{b}}$ & $45.7^{\mathrm{b}}$ & $48.0^{\mathrm{b}}$ & $47.2^{\mathrm{b}}$ & $52.1^{\mathrm{c}}$ & $54.0^{\mathrm{c}}$ & $57.1^{\mathrm{c}}$ & $59.3^{\mathrm{c}}$ & $64.2^{\mathrm{c}}$ \\
\hline $\mathrm{CC}$ & 0 & 0 & $21.0^{\mathrm{b}}$ & $26.2^{\mathrm{b}}$ & $32.0^{\mathrm{b}}$ & $35.1^{\mathrm{b}}$ & $39.4^{\mathrm{b}}$ & $42.5^{\mathrm{b}}$ & $44.1^{\mathrm{b}}$ & $46.9^{\mathrm{b}}$ & $48.0^{\mathrm{b}}$ & $51.3^{\mathrm{c}}$ & $53.0^{\mathrm{c}}$ & $52.8^{\mathrm{c}}$ \\
\hline CG & 0 & 0 & $5.2^{\mathrm{a}}$ & $22.4^{\mathrm{b}}$ & $33.1^{\mathrm{b}}$ & $38.2^{\mathrm{b}}$ & $43.0^{\mathrm{b}}$ & $45.6^{\mathrm{b}}$ & $47.0^{\mathrm{b}}$ & $49.5^{\mathrm{c}}$ & $52.2^{\mathrm{c}}$ & $54.0^{\mathrm{c}}$ & $56.1^{\mathrm{c}}$ & $58.8^{\mathrm{c}}$ \\
\hline SB & 0 & 0 & $49.8^{\mathrm{c}}$ & $53.8^{\mathrm{c}}$ & $55.0^{\mathrm{c}}$ & $58.9^{\mathrm{b}}$ & $60.0^{\mathrm{c}}$ & $63.1^{\mathrm{b}}$ & $64.0^{\mathrm{c}}$ & $67.1^{\mathrm{c}}$ & $69.4^{\mathrm{c}}$ & $73.0^{\mathrm{d}}$ & $74.8^{\mathrm{d}}$ & $75.5^{\mathrm{d}}$ \\
\hline \multicolumn{15}{|c|}{ Week 36} \\
\hline C R & 0 & 0 & $18.3^{\mathrm{a}}$ & $25.0^{\mathrm{b}}$ & $28.8^{\mathrm{b}}$ & $30.5^{\mathrm{b}}$ & $33.8^{\mathrm{b}}$ & $37.5^{\mathrm{b}}$ & $41.1^{\mathrm{b}}$ & $44.6^{\mathrm{b}}$ & $47.0^{\mathrm{b}}$ & $51.2^{\mathrm{c}}$ & $54.4^{\mathrm{c}}$ & $56.1^{\mathrm{c}}$ \\
\hline E S & 0 & 0 & $25.2^{\mathrm{b}}$ & $27.0^{\mathrm{b}}$ & $35.8^{\mathrm{b}}$ & $45.7^{\mathrm{b}}$ & $55.1^{\mathrm{c}}$ & $54.0^{\mathrm{b}}$ & $57.8^{\mathrm{c}}$ & $61.2^{\mathrm{c}}$ & $62.0^{\mathrm{c}}$ & $63.9^{\mathrm{c}}$ & $65.1^{\mathrm{c}}$ & $65.2^{\mathrm{c}}$ \\
\hline EM & 0 & 0 & $30.0^{\mathrm{b}}$ & $33.2^{\mathrm{b}}$ & $36.1^{\mathrm{b}}$ & $39.2^{\mathrm{b}}$ & $42.9^{\mathrm{b}}$ & $44.9^{\mathrm{b}}$ & $47.6^{\mathrm{b}}$ & $51.4^{\mathrm{c}}$ & $55.5^{\mathrm{c}}$ & $58.8^{\mathrm{b}}$ & $62.4^{\mathrm{c}}$ & $68.8^{\mathrm{c}}$ \\
\hline $\mathrm{CC}$ & 0 & 0 & $23.8^{\mathrm{b}}$ & $27.9^{\mathrm{b}}$ & $30.7^{b}$ & $37.7^{\mathrm{b}}$ & $41.2^{\mathrm{b}}$ & $44.4^{\mathrm{b}}$ & $47.3^{\mathrm{b}}$ & $51.6^{\mathrm{c}}$ & $53.9^{c}$ & $55.6^{\mathrm{b}}$ & $58.8^{\mathrm{c}}$ & $62.0^{\mathrm{c}}$ \\
\hline $\mathrm{CG}$ & 0 & 0 & $7.0^{\mathrm{a}}$ & $22.1^{\mathrm{b}}$ & $31.8^{\mathrm{b}}$ & $36.4^{\mathrm{b}}$ & $41.0^{c}$ & $44.2^{\mathrm{b}}$ & $45.0^{\mathrm{b}}$ & $48.6^{\mathrm{b}}$ & $52.7^{\mathrm{c}}$ & $55.0^{\mathrm{b}}$ & $61.1^{\mathrm{c}}$ & $63.2^{\mathrm{c}}$ \\
\hline SB & 0 & 0 & $52.9^{c}$ & $61.0^{\mathrm{c}}$ & $63.1^{\mathrm{c}}$ & $64.1^{\mathrm{c}}$ & $66.1^{\mathrm{c}}$ & $68.2^{\mathrm{c}}$ & $68.9^{c}$ & $71.0^{\mathrm{d}}$ & $72.4^{\mathrm{d}}$ & $74.5^{\mathrm{d}}$ & $75.1^{\mathrm{d}}$ & $76.1^{d}$ \\
\hline
\end{tabular}

Means within the same columns with different superscripts are significantly different at $\mathrm{p}<0.05$.

Key: $C R=$ Chloris roxburghiana, $E S=$ Eragrostis superba, $E M=$ Enteropogon macrostachyus, $C C=$ Cenchrus ciliaris, $C G=$ Chloris gayana, $S B=$ Sorghum sudanense 
Table 4. Daily \% seed germination of six range grass species after storage period of 2, 12, 24 and 36 weeks from DOH, produced under rain fed treatment (Control)

\begin{tabular}{|c|c|c|c|c|c|c|c|c|c|c|c|c|c|c|}
\hline Days & 1 & 2 & 3 & 4 & 5 & 6 & 7 & 8 & 9 & 10 & 11 & 12 & 13 & 14 \\
\hline \multicolumn{15}{|c|}{ Week 2} \\
\hline C R & 0 & 0 & 0 & 0 & 0 & 0 & 0 & 0 & 0 & 0 & 0 & 0 & 0 & 0 \\
\hline ES & 0 & 0 & 0 & 0 & 0 & 0 & 0 & 0 & 0 & 0 & 0 & 0 & 0 & 0 \\
\hline EM & 0 & 0 & 0 & 0 & 0 & 0 & 0 & 0 & $3.0^{\mathrm{a}}$ & $3.0^{\mathrm{a}}$ & $4.2^{\mathrm{a}}$ & $5.2^{\mathrm{a}}$ & $6.3^{\mathrm{a}}$ & $7.5^{\mathrm{a}}$ \\
\hline $\mathrm{CC}$ & 0 & 0 & 0 & 0 & 0 & 0 & 0 & 0 & 0 & 0 & 0 & 0 & 0 & 0 \\
\hline $\mathrm{CG}$ & 0 & 0 & 0 & 0 & 0 & 0 & 0 & $3.0^{\mathrm{a}}$ & $6.5^{\mathrm{a}}$ & $7.7^{\mathrm{a}}$ & $8.5^{\mathrm{a}}$ & $11.1^{\mathrm{a}}$ & $12.0^{\mathrm{a}}$ & $12.0^{\mathrm{a}}$ \\
\hline SB & 0 & 0 & $2.1^{\mathrm{a}}$ & $3.1^{\mathrm{a}}$ & $5.5^{\mathrm{a}}$ & $9.8^{\mathrm{a}}$ & $14.2^{\mathrm{a}}$ & $14.6^{\mathrm{a}}$ & $18.2^{\mathrm{a}}$ & $25.2^{\mathrm{b}}$ & $28.8^{\mathrm{b}}$ & $32.0^{\mathrm{b}}$ & $36.1^{\mathrm{b}}$ & $48.2^{\mathrm{c}}$ \\
\hline \multicolumn{15}{|c|}{ Week 12} \\
\hline C R & 0 & 0 & $2.2^{\mathrm{a}}$ & $3.0^{\mathrm{a}}$ & $6.0^{\mathrm{a}}$ & $8.2^{\mathrm{a}}$ & $11.0^{\mathrm{a}}$ & $12.3^{\mathrm{a}}$ & $12.5^{\mathrm{a}}$ & $13.4^{\mathrm{a}}$ & $14.2^{\mathrm{a}}$ & $14.0^{\mathrm{a}}$ & $16.0^{\mathrm{a}}$ & $17.2^{\mathrm{a}}$ \\
\hline ES & 0 & 0 & $4.0^{\mathrm{a}}$ & $8.3^{\mathrm{a}}$ & $9.0^{\mathrm{a}}$ & $9.1^{\mathrm{a}}$ & $9.8^{\mathrm{a}}$ & $11.4^{\mathrm{a}}$ & $12.7^{\mathrm{a}}$ & $13.0^{\mathrm{a}}$ & $13.1^{\mathrm{a}}$ & $13.0^{\mathrm{a}}$ & $16.0^{\mathrm{a}}$ & $18.5^{\mathrm{a}}$ \\
\hline EM & 0 & 0 & $7.0^{\mathrm{a}}$ & $11.1^{\mathrm{a}}$ & $14.2^{\mathrm{a}}$ & $17.3^{\mathrm{a}}$ & $18.2^{\mathrm{a}}$ & $20.4^{\mathrm{b}}$ & $21.0^{\mathrm{a}}$ & $24.0^{\mathrm{b}}$ & $25.8^{\mathrm{b}}$ & $29.5^{\mathrm{b}}$ & $34.0^{\mathrm{b}}$ & $39.5^{\mathrm{b}}$ \\
\hline $\mathrm{CC}$ & 0 & 0 & $3.0^{\mathrm{a}}$ & $6.5^{\mathrm{a}}$ & $7.5^{\mathrm{a}}$ & $11.1^{\mathrm{a}}$ & $11.8^{\mathrm{a}}$ & $13.0^{\mathrm{a}}$ & $13.0^{\mathrm{a}}$ & $14.0^{\mathrm{a}}$ & $14.5^{\mathrm{a}}$ & $19.0^{\mathrm{a}}$ & $28.1^{\mathrm{b}}$ & $30.0^{\mathrm{b}}$ \\
\hline $\mathrm{CG}$ & 0 & 0 & $5.1^{\mathrm{a}}$ & $6.0^{\mathrm{a}}$ & $13.0^{\mathrm{a}}$ & $16.4^{\mathrm{a}}$ & $20.1^{\mathrm{a}}$ & $24.2^{\mathrm{b}}$ & $24.4^{\mathrm{b}}$ & $28.4^{\mathrm{b}}$ & $33.1^{\mathrm{b}}$ & $37.0^{\mathrm{b}}$ & $41.5^{\mathrm{b}}$ & $47.0^{\mathrm{c}}$ \\
\hline SB & 0 & 0 & $14.5^{\mathrm{a}}$ & $27.0^{\mathrm{b}}$ & $28.1^{\mathrm{b}}$ & $32.4^{\mathrm{b}}$ & $33.0^{\mathrm{b}}$ & $37.0^{\mathrm{b}}$ & $42.3^{\mathrm{b}}$ & $45.0^{\mathrm{b}}$ & $45.3^{\mathrm{b}}$ & $48.0^{\mathrm{c}}$ & $50.3^{\mathrm{c}}$ & $55.1^{\mathrm{c}}$ \\
\hline \multicolumn{15}{|c|}{ Week 24} \\
\hline C R & 0 & 0 & $2.8^{\mathrm{a}}$ & $6.3^{\mathrm{a}}$ & $9.2^{\mathrm{a}}$ & $12.3^{\mathrm{a}}$ & $24.0^{\mathrm{b}}$ & $30.1^{\mathrm{b}}$ & $34.0^{\mathrm{b}}$ & $38.0^{\mathrm{b}}$ & $42.0^{\mathrm{b}}$ & $44.2^{\mathrm{b}}$ & $46.7^{\mathrm{b}}$ & $49.3^{\mathrm{c}}$ \\
\hline E S & 0 & 0 & $10.2^{\mathrm{a}}$ & $19.3^{\mathrm{a}}$ & $22.0^{\mathrm{b}}$ & $28.0^{\mathrm{b}}$ & $37.2^{\mathrm{b}}$ & $42.0^{\mathrm{b}}$ & $45.1^{\mathrm{b}}$ & $49.1^{\mathrm{c}}$ & 51.2 & $52.0^{\mathrm{c}}$ & $50.4^{\mathrm{c}}$ & $52.5^{\mathrm{c}}$ \\
\hline EM & 0 & 0 & $14.3^{\mathrm{a}}$ & $21.2^{\mathrm{b}}$ & $28.1^{\mathrm{b}}$ & $37.0^{\mathrm{b}}$ & $43.1^{\mathrm{b}}$ & $45.0^{\mathrm{b}}$ & $45.2^{\mathrm{b}}$ & $47.0^{\mathrm{c}}$ & 49.3 & $52.0^{\mathrm{c}}$ & $54.1^{\mathrm{c}}$ & $54.2^{\mathrm{c}}$ \\
\hline $\mathrm{CC}$ & 0 & 0 & $18.0^{\mathrm{a}}$ & $21.7^{\mathrm{b}}$ & $27.0^{\mathrm{b}}$ & $34.2^{\mathrm{b}}$ & $35.2^{\mathrm{b}}$ & $40.5^{\mathrm{b}}$ & $43.6^{\mathrm{b}}$ & $47.3^{\mathrm{c}}$ & 47.0 & $47.3^{\mathrm{c}}$ & $47.5^{\mathrm{c}}$ & $49.5^{\mathrm{c}}$ \\
\hline $\mathrm{CG}$ & 0 & 0 & $5.9^{\mathrm{a}}$ & $14.6^{\mathrm{a}}$ & $24.5^{\mathrm{b}}$ & $29.6^{\mathrm{b}}$ & $33.0^{\mathrm{b}}$ & $38.6^{\mathrm{b}}$ & $41.1^{\mathrm{b}}$ & $45.5^{\mathrm{c}}$ & 46.2 & $47.0^{\mathrm{c}}$ & $46.1^{\mathrm{b}}$ & $52.0^{\mathrm{c}}$ \\
\hline SB & 0 & 0 & $33.1^{\mathrm{b}}$ & $38.0^{\mathrm{b}}$ & $42.0^{\mathrm{b}}$ & $48.7^{\mathrm{c}}$ & $52.0^{\mathrm{c}}$ & $54.0^{\mathrm{c}}$ & $54.0^{\mathrm{b}}$ & $57.2^{\mathrm{c}}$ & $57.0^{c}$ & $59.1^{\mathrm{d}}$ & $62.0^{\mathrm{d}}$ & $65.3^{\mathrm{d}}$ \\
\hline \multicolumn{15}{|c|}{ Week 36} \\
\hline $\mathrm{C} \mathrm{R}$ & 0 & 0 & $14.8^{\mathrm{a}}$ & $18.7^{\mathrm{a}}$ & $23.8^{\mathrm{b}}$ & $29.0^{\mathrm{b}}$ & $31.0^{\mathrm{b}}$ & $34.2^{\mathrm{b}}$ & $38.1^{\mathrm{b}}$ & $42.0^{\mathrm{b}}$ & $44.0^{\mathrm{b}}$ & $47.2^{\mathrm{c}}$ & $48.4^{\mathrm{c}}$ & $51.0^{\mathrm{c}}$ \\
\hline E S & 0 & 0 & $19.8^{\mathrm{a}}$ & $20.4^{\mathrm{b}}$ & $27.0^{\mathrm{b}}$ & $35.2^{\mathrm{b}}$ & $35.3^{\mathrm{b}}$ & $41.0^{\mathrm{b}}$ & $45.9^{\mathrm{b}}$ & $49.7^{\mathrm{c}}$ & $51.0^{\mathrm{c}}$ & $53.6^{\mathrm{c}}$ & $55.0^{\mathrm{c}}$ & $55.7^{\mathrm{c}}$ \\
\hline EM & 0 & 0 & $24.6^{\mathrm{b}}$ & $25.2^{\mathrm{b}}$ & $34.2^{\mathrm{b}}$ & $38.0^{\mathrm{b}}$ & $40.0^{\mathrm{b}}$ & $43.9^{\mathrm{b}}$ & $47.0^{\mathrm{c}}$ & $50.1^{\mathrm{c}}$ & $52.6^{\mathrm{c}}$ & $55.0^{\mathrm{c}}$ & $55.4^{\mathrm{c}}$ & $58.8^{\mathrm{c}}$ \\
\hline $\mathrm{CC}$ & 0 & 0 & $23.4^{\mathrm{b}}$ & $23.0^{\mathrm{b}}$ & $29.7^{\mathrm{b}}$ & $38.4^{\mathrm{b}}$ & $44.1^{\mathrm{b}}$ & $45.0^{\mathrm{b}}$ & $48.2^{\mathrm{c}}$ & $53.7^{\mathrm{c}}$ & $53.9^{\mathrm{c}}$ & $54.0^{\mathrm{c}}$ & $55.8^{\mathrm{c}}$ & $57.0^{\mathrm{c}}$ \\
\hline $\mathrm{CG}$ & 0 & 0 & $6.5^{\mathrm{a}}$ & $19.3^{\mathrm{a}}$ & $29.0^{\mathrm{b}}$ & $38.0^{\mathrm{b}}$ & $44.0^{\mathrm{b}}$ & $44.6^{\mathrm{b}}$ & $49.0^{\mathrm{c}}$ & $49.6^{\mathrm{c}}$ & $48.0^{\mathrm{c}}$ & $51.3^{\mathrm{c}}$ & $55.6^{\mathrm{c}}$ & $61.2^{\mathrm{d}}$ \\
\hline SB & 0 & 0 & $45.8^{\mathrm{b}}$ & $50.3^{\mathrm{c}}$ & $50.8^{\mathrm{c}}$ & $52.2^{\mathrm{c}}$ & $53.7^{\mathrm{c}}$ & $56.0^{\mathrm{c}}$ & $57.0^{\mathrm{c}}$ & $57.9^{\mathrm{c}}$ & $62.4^{\mathrm{d}}$ & $64.5^{\mathrm{d}}$ & $65.1^{\mathrm{d}}$ & $69.5^{\mathrm{d}}$ \\
\hline
\end{tabular}

Means within the same columns with different superscripts are significantly different at $\mathrm{p}<0.05$.

Key: $C R=$ Chloris roxburghiana, $E S=$ Eragrostis superba, $E M=$ Enteropogon macrostachyus, $C C=$ Cenchrus ciliaris, $C G=$ Chloris gayana, $S B=$ Sorghum sudanense

\subsection{Germination Index (GI)}

Germination index for the grasses are presented in table 5. There was no significant difference in germination rates for specific species across the different soil water content levels of 80,50 and $30 \% \mathrm{FC}$ and the rain fed condition. Seeds produced under rain fed conditions had lower GI but not significantly different from watered conditions. There was significant difference $(\mathrm{p} \leq 0.05)$ in GI for the specific species with storage period $(2,12,24$ and 36 weeks from DOH) for five grasses except Sorghum sudanense. The general trend observed was an increase in GI with storage period. Sorghum sudanense showed higher GI $(>20)$ across all the soil water content levels. However, under rain fed treatment the GI was $<20$ except after storage duration of 36 weeks which was not significant different from the other storage periods. After storage period of 
12 weeks onwards, the GI of Sorghum sudanense were higher, followed by Enteropogon macrostachyus and Cenchrus ciliaris.

These findings suggest storage period of grass seeds influences germination and hence viability. The observed significant differences $(p \leq 0.05)$ on grass seeds germination after different storage periods provides insights on understanding the agronomic practices that informs farmers on harvesting, storage and the optimum use periods for reseeding or establishment of pastures. Similar findings were reported by Mganga [18] who found out freshly harvested seeds of Enteropogon macrostachyus, Cenchrus ciliaris and Eragrostis superba failed to germinate under laboratory tests. However, the same seeds were tested for germination after two years storage and observed to germinate under the same condition. This observation relate to the observations of Chloris roxburghiana and Cenchrus ciliaris in this study which failed to germinate after two weeks of storage from DOH but at 36 weeks storage period, showed over $50 \%$ percent germination.

Table 5. Germination Index of six range grass species after storage period of 2, 12,24 and 36 weeks from date of harvest, produced at $80,50,30 \% \mathrm{FC}$ soil water content and Rain-fed

\begin{tabular}{|c|c|c|c|c|}
\hline & $80 \% \mathrm{FC}$ & $50 \% \mathrm{FC}$ & $30 \% \mathrm{FC}$ & Rain-fed (control) \\
\hline \multicolumn{5}{|c|}{ Week 2} \\
\hline C R & 0.0 & 0.0 & 0.0 & 0.0 \\
\hline ES & $0.2^{\mathrm{a}}$ & 0.0 & $1.1^{\mathrm{a}}$ & $1.0^{\mathrm{a}}$ \\
\hline EM & $3.1^{\mathrm{a}}$ & $2.7^{\mathrm{a}}$ & $2.0^{\mathrm{a}}$ & $1.5^{\mathrm{a}}$ \\
\hline $\mathrm{CC}$ & 0.0 & $0.1^{\mathrm{a}}$ & 0.0 & 0.0 \\
\hline $\mathrm{CG}$ & $2.4^{\mathrm{a}}$ & $1.8^{\mathrm{a}}$ & $2.1^{\mathrm{a}}$ & $1.7^{\mathrm{a}}$ \\
\hline SB & $25.3^{\mathrm{b}}$ & $28.4^{\mathrm{b}}$ & $24.3^{\mathrm{a}}$ & $13.4^{\mathrm{a}}$ \\
\hline \multicolumn{5}{|c|}{ Week 12} \\
\hline C R & $6.4^{\mathrm{a}}$ & $8.1^{\mathrm{a}}$ & $8.8^{\mathrm{a}}$ & $3.4^{\mathrm{a}}$ \\
\hline E S & $12.4^{\mathrm{a}}$ & $16.3^{\mathrm{a}}$ & $11.5^{\mathrm{a}}$ & $8.8^{\mathrm{a}}$ \\
\hline EM & $21.6^{\mathrm{b}}$ & $27.5^{\mathrm{b}}$ & $23.2^{\mathrm{b}}$ & $12.5^{\mathrm{a}}$ \\
\hline $\mathrm{CC}$ & $14.2^{\mathrm{a}}$ & $15.1^{\mathrm{a}}$ & $18.3^{\mathrm{a}}$ & $6.8^{\mathrm{a}}$ \\
\hline $\mathrm{CG}$ & $12.5^{\mathrm{a}}$ & $15.7^{\mathrm{a}}$ & $16.8^{\mathrm{a}}$ & $10.2^{\mathrm{a}}$ \\
\hline SB & $27.7^{\mathrm{b}}$ & $31.6^{\mathrm{b}}$ & $26.5^{\mathrm{b}}$ & $18.8^{\mathrm{a}}$ \\
\hline \multicolumn{5}{|c|}{ Week 24} \\
\hline C R & $8.5^{\mathrm{a}}$ & $8.6^{\mathrm{a}}$ & $9.4^{\mathrm{a}}$ & $5.5^{\mathrm{a}}$ \\
\hline E S & $13.1^{\mathrm{a}}$ & $15.5^{\mathrm{a}}$ & $14.2^{\mathrm{a}}$ & $10.3^{\mathrm{a}}$ \\
\hline EM & $20.9^{b}$ & $24.3^{\mathrm{b}}$ & $22.8^{\mathrm{b}}$ & $12.5^{\mathrm{a}}$ \\
\hline $\mathrm{CC}$ & $18.6^{\mathrm{a}}$ & $21.2^{\mathrm{b}}$ & $24.4^{\mathrm{b}}$ & $15.2^{\mathrm{a}}$ \\
\hline $\mathrm{CG}$ & $15.2^{\mathrm{a}}$ & $13.8^{\mathrm{a}}$ & $16.1^{\mathrm{a}}$ & $13.4^{\mathrm{a}}$ \\
\hline SB & $25.2^{\mathrm{b}}$ & $26.3^{\mathrm{b}}$ & $24.8^{\mathrm{b}}$ & $18.3^{\mathrm{a}}$ \\
\hline \multicolumn{5}{|c|}{ Week 36} \\
\hline C R & $8.8^{\mathrm{a}}$ & $7.3^{\mathrm{a}}$ & $9.2^{\mathrm{a}}$ & $7.5^{\mathrm{a}}$ \\
\hline E S & $14.1^{\mathrm{a}}$ & $13.9^{\mathrm{a}}$ & $12.6^{\mathrm{a}}$ & $11.3^{\mathrm{a}}$ \\
\hline EM & $22.3^{\mathrm{b}}$ & $25.1^{\mathrm{b}}$ & $23.7^{\mathrm{b}}$ & $17.1^{\mathrm{a}}$ \\
\hline $\mathrm{CC}$ & $19.1^{\mathrm{a}}$ & $18.6^{\mathrm{a}}$ & $24.7^{\mathrm{b}}$ & $15.2^{\mathrm{a}}$ \\
\hline CG & $14.8^{\mathrm{a}}$ & $15.9^{\mathrm{a}}$ & $15.8^{\mathrm{a}}$ & $12.9^{\mathrm{a}}$ \\
\hline SB & $25.2^{\mathrm{b}}$ & $23.6^{\mathrm{b}}$ & $27.2^{\mathrm{b}}$ & $21.1^{\mathrm{b}}$ \\
\hline
\end{tabular}

Means within the same columns with different superscripts are significantly different at $\mathrm{p}<0.05$.

Key: $C R=$ Chloris roxburghiana, $E S=$ Eragrostis superba, $E M=$ Enteropogon macrostachyus, $C C=$ Cenchrus ciliaris, $C G=$ Chloris gayana, $S B=$ Sorghum sudanense 


\section{Discussions}

The observed no effects of the three soil water content on seed quality should not be the only factor in consideration for seed production, this is so since soil water content had effect on seed quantity yields which is critical for pasture productivity. Range grass seeds for reseeding should be produced in adequate quantities and better quality if sustainable pasture management is to be maintained [15]. Lusembo [16] reported that many East African drylands could not depend on imported grass seeds under the low local seed production challenges, hence the need for pasture seed production intensification. The observed higher germination percentage for Chloris gayana and Sorghum sudanense provides a greater opportunity for pasture seed production under the current declining water for irrigation, which is further threatened by climate change phenomenon.

The observed increase in GP of Cenchrus ciliaris, Enteropogon macrostachyus, Chloris roxburghiana, Eragrostis superba and Chloris gayana which attained germination $>50 \%$ by week 24 gives in-depth insights on the need to avoid use of freshly harvested seeds of these grasses. The high GP observed in Sorghum sudanense at early stages can be related to the large seed size compared to the other five species [11]. Dourado [6] reported seed size to have direct bearing on seed quality and established a positive correlation between seed size and germination percent. The higher germination percent observed in Enteropogon macrostachyus over Cenchrus ciliaris and Eragrostis superba can be attributed to the know seed dormancy related to integument only for Enteropogon macrostachyus, with the latter having both integument and embryo dormancy especially at early storage period [17].

Faster seed germination is desirable for range grasses under field conditions, as this allow seeds to germinate and establish faster during the initial precipitation received, hence increasing chances of survival if dry spell follows [18]. This can explain the higher establishment and subsequent higher biomass yields observed for Sorghum sudanense and Chloris gayana ( 964.8 and $832.7 \mathrm{Kg} / \mathrm{ha}$, respectively) in this study under rain fed conditions.

The observed higher percent germination of Chloris gayana over Cenchrus ciliaris in this study confirms the sentiments by Dourado [6] that some grass species can have higher germination percent but low germination rates. Germination percentage is a measure of seed quality [13], however, the seeds vigour and the rates of germination are other important measure of seed quality and can be evaluated through GI tests $[4,16]$.

The findings showed grass species to have similar germination percent but varied germination index for some species. This was observed in Sorghum sudanense, Chloris gayana and Enteropogon macrostachyus which attained higher germination index within 4 days of incubation while Chloris roxburghiana, Cenchrus ciliaris and Eragrostis superba attaining higher germination index at over 7 days incubation periods. Dourado [6] also reported that seeds may have same germination percent but the rates of germination are different. Seed that germinate faster have an advantage of head start and hence reduce competition from fast germinating weeds as well as avoiding moisture stress in the dry rangelands [17,20,2235]. Sorghum sudanense and Enteropogon macrostachyus showed higher germination rates which can be attributed to seed size and dormancy mechanism which were relatively better compared to the other four species.

The observed higher GI for Cenchrus ciliaris over Chloris gayana can be attributed to the presence of hairy fascicles that reduced moisture loss around the caryopsis of Cenchrus ciliaris, hence enhancing germination rates. This was also reported for the same species by $[5,20,38]$ where Cencrus ciliaris had higher germination rates than Eragrostis superba. This finding suggests that individual grass species have different germination rates, which could be attributed to plants adaptive strategies to survival under the harsh and unpredictable climates through either morphological or physiological responses $[29,27]$.

The observed low germination rate for Cenchrus ciliaris under rainfed treatment compared to watered condition suggests an imposed dormancy by water stress during seed maturation. This was observed from the dry seasons that prevailed during seed setting under rainfed condition lasting for the whole seeding period and hence probable reason of the dormancy. This finding concurs with Sharif-Zadeh and Murdoch [33] working with Cenchrus ciliaris and reported seed dormancy to increase when water stress is imposed during maturation, especially when mother plants are exposed to water stress after caryopses are fully matured. This was observed in this study where maturity and heading of the grasses occurred when rains had ceased and the species matured and seeded under dry season.

These findings on percent seed germination and germination index for the six grass species under rainfed conditions closely explains the normally observed performance of these grasses under natural condition. Sorghum sudanense, Enteropogon macrostachyus had higher performance in terms of biomass yields and also emerged with higher germination percent. This represents the true responses under natural field condition for the grass species with better GP and GI being dominant over others. The same species also get added advantages when grown under irrigation where water stresses are reduced.

\section{Conclusion and Recommendation}

The results have shown that storage period of more than 12 weeks gives better germination percent and germination index for all the six grass species. Sorghum sudanense showed better adaptation to germination at early storage period of 2 weeks by having $>50 \%$ germination percent at week 2. Enteropogon macrostachyus and Chloris gayana showed lower $(<15 \%)$ germination percent at week 2 . Eragrostis superba, Cenchrus ciliaris, and Chloris 
roxburghiana did not germinate after 2 weeks of storage. It is therefore recommended that;

1. Farmers should not use freshly harvested seeds of Eragrostis superba, Cenchrus ciliaris, and Chloris roxburghiana to reseed or establish pastures, at least 12 weeks storage duration is required.

2. Sorghum sudanense and Enteropogon macrostachyus have early germination abilities but also needs at least 12 weeks storage period for increased germination rates and germination percentage.

3. Irrigation at the three soil water content does not affect germination percentage and germination index.

\section{Acknowledgements}

This work was supported by National Irrigation Board (NIB) Kenya, National Council of Science, Technology and Innovation (NCSTI), German Academic Exchange Service (DAAD)-Kenya and the University of Nairobi, Kenya

\section{REFERENCE}

[1] Awad, A., Hafiz, S., Hammada, M.S, El-Nouby, A., \& El-Hendawy, S. (2013) Grain yield production of Sorghum sudanense (Sudan grass) as influenced by cutting numbers, potassium rates, and intrarow spacing in a semiarid environment. Turkish Journal of Agriculture and Forestry, $37(6), 657-664$

[2] Bestelmeyer, B.T., Miller, J.R. \& Wiens, J.A. (2003) Applying species diversity theory to land management. Ecological Applications, 13(6), 1750-1761.

[3] Boonman, G, (1993) (ed) East Africa's grasses and fodders: Their ecology and husbandry (Vol. 29). Springer.

[4] Chin, H.F. \& Wong, C.C. (1993). The importance of tropical pasture seed quality and factors affecting it in smallholder farming systems. In Proc. XVII Int. Grassland Congress.

[5] Dewir, Y.H., El-Mahrouk, M.E., \& Naidoo, Y. (2011) Effect of some mechanical and chemical treatments on seed germination of Sabal palmetto and Thrinax morrisii palms. Australian Journal of Crop Science, 5(3), 248-253

[6] Dourado, A.M. (1989) The effect of seed size, method of harvest and accelerated ageing on germination. Proceedings of the International Seed Test Association, 17. http://agris.fao.org/agris-search/search/display.do?f=1989/C H/CH89002.xml;CH8923236

[7] Facelli, J.M., Chesson, P. \& Barnes, N. (2005) Differences in seed biology of annual plants in arid lands: a key ingredient of the storage effect. Ecology, 86(11), 2998-3006.

[8] Fynn, R.W. (2012) Functional resource heterogeneity increases livestock and rangeland productivity. Rangeland Ecology \& Management, 65(4), 319-329.

[9] GOK, (2005) Tana River District Strategic Plan 2005-2010 for Implementation of the National Population Policy for Sustainable Development
http://ncpdnke.org/strategicplans/TanaRiver.pdf. (Accessed on 25 June 2012).

[10] Griffiths, R., (1993) Sustainable supplies of quality herbage seed for sub-Saharan Africa smallholder's pp. 662-665. In: MJ Baker (ed). Grasslands for our world. Sir Publishing, Wellington, New Zealand

[11] Guo, Q., Brown, J.H., Valone, T.J. \& Kachman, S.D. (2000) Constraints of seed size on plant distribution and abundance. Ecology, 81(8), 2149-2155.

[12] ISTA. (1987) Handbook of Vigour Test Methods." International Seed Testing Association"

[13] ISTA. (1976) International rules for seed testing. Seed Science and Technology 4:23-28

[14] Kreuter, U.P., Fox, W.E., Tanaka, J.A., Maczko, K.A., McCollum, D.W., Mitchell, J.E., \& Hidinger, L. (2012) Framework for comparing ecosystem impacts of developing unconventional energy resources on western US rangelands. Rangeland Ecology and Management, 65(5), 433-443.

[15] Loch, D.S. (1985) Commercial seed increase of new pasture cultivars: Organization and Practice. In: Kategile J.A. (ed), Pasture Improvement Research in Eastern and Southern Africa. IDRC-273e. Proceedings of a workshop held at Harare, Zimbabwe, 17-21 September 1984.

[16] Lusembo, P., Sabiiti, E.N., Mugerwa, J.S., Ndikumana, J. \& Leeuw, P.D. (1993). The effect of staking on seed yield and quality characteristics of Macroptilium atropurpureum at Namulonge, Uganda. In Workshop for the African Feed Resources Network (AFRNET), Harare (Zimbabwe) 6-10 December 1993.

[17] Mganga, K. Z., Musimba, N.K., Nyangito, M.M., Nyariki, D.M., Mwangombe, A.W., Ekaya, W.N. \& Verhagen, J. (2010a). The role of moisture in the successful rehabilitation of denuded patches of a semi-arid environment in Kenya. Journal of Environmental Science and Technology, 3, 195-207.

[18] Mganga, K.Z., Musimba, N.K., Nyariki, D.M., Nyangito, M.M., Mwang'ombe, A.W., Ekaya, W.N. \& Muiru, W.M. (2010b). Dry matter yields and hydrological properties of three perennial grasses of a semi-arid environment in east Africa. African journal of Plant Science, 4(5), 138-144

[19] Mganga, K.Z., Musimba, N.R., Nyariki, D.M., Nyangito, M.M. \& Mwang'ombe, A.W. (2013). The choice of grass species to combat desertification in semi-arid Kenyan rangelands is greatly influenced by their forage value for livestock. Grass and Forage Science.

[20] Mganga, K.Z. (2009). Impact of grass reseeding technology on rehabilitation of the degraded rangelands: a case study of Kibwezi District, Kenya. MSc Thesis, University of Nairobi, Nairobi, Kenya

[21] Mlote, S.N., Mdoe, N.S., Isinika, A.C. \& Mtenga, L.A. (2013). Factors Affecting Agro-Pastoralist and Pastoralists' Willingness to Adopt Beef Cattle Fattening in the Lake Zone in Tanzania. Journal of Agricultural Science (1916-9752), $5(10)$.

[22] Mnene, W.N. (2005). Strategies to increase success rates in natural pasture development through reseeding degraded rangelands of Kenya. PhD Thesis, University of Nairobi, Nairobi, Nairobi, Kenya. 
[23] Musimba, N.K.R., Nyariki, D.M., Ikutwa, C.N. \& Teka, T. (2004) Dryland Husbandry for sustainable development in the southern rangelands of Kenya. OSSREA, Addis Ababa, 2004.

[24] Mwadalu, R. \& Mwangi, M. (2013) The potential role of sorghum in enhancing food security in semi-arid eastern Kenya: A review. Journal of Applied Biosciences, 71(1), 5786-5799.

[25] Nyangito, M.M., Musimba, N.K.R. \& Nyariki, D.M. (2008). Range use and dynamics in the agropastoral system of southeastern Kenya. African Journal of Environmental Science and Technology 2(8): 220-230

[26] Nyangito, M.M., Musimba, N.K.R. \& Nyariki, D.M. (2009) Hydrological properties of grazed perennial swards in semiarid southeastern Kenya. African Journal of Environmental Science and Technology 3(2): 026-033.

[27] Opiyo, F.O. (2007) Land treatment effects on morphometric characteristics of three grass species and economic returns from reseeding in Kitui district, Kenya. MSc Thesis, University of Nairobi, Nairobi, Kenya.

[28] Pizarro, E.A., Hare, M.D., Mutimura, M. \& Changjun, B. (2013) Brachiaria hybrids: potential, forage use and seed yield. Tropical Grasslands-Forrajes Tropicales, 1(1), 31-35.

[29] Qi, M.Q. \& Redmann, R.E. (1993) Seed germination and seedling survival of $\mathrm{C} 3$ and $\mathrm{C} 4$ grasses under water stress. Journal of Arid Environments, 24(3), 277-285.

[30] Rietkerk, M., Ketner, P., Burger, J., Hoorens, B. \& Olff, H. (2000) Multiscale soil and vegetation patchiness along a gradient of herbivore impact in a semi-arid grazing system in West Africa. Plant Ecology, 148:207-224.

[31] Samuel, T. (2013). From cattle herding to sedentary agriculture: the role of hamer women in the transition. African study monographs. Supplementary issue., 46, 121-133.

[32] Seobi, T., Anderson, S.H., Udawatta, R.P. \& Gantzer, C.J. (2005) Influence of grass and agroforestry buffer strips on soil hydraulic properties for an Albaqualf. Soil Science Society of America Journal 69:893-901.

[33] Sharif-Zadeh, F \& Murdoch, A. J. (2000) The effects of different maturation conditions on seed dormancy and germination of Cenchrus ciliaris. Seed Science Research, 10(04), 447-457.

[34] Tarawali, S.A., Tarawali, G., Larbi, A. \& Hanson, J. (1995) Methods for the Evaluation of Legumes Grasses and Fodder Trees for Use as Livestock Feed. ILRI Manual 1. ILRI (International Livestock Research Institute), Nairobi, Kenya. pp. 51.

[35] Verdú, M. \& Traveset, A., (2005) Early emergence enhances plant fitness: a phylogenetically controlled meta-analysis. Ecology, 86(6), 1385-1394.

[36] Visser, N., Morris, C., Hardy, M.B. \& Botha, J.C, (2007) Restoring bare patches in the Nama-Karoo of South Africa. African Journal of Range and Forage Science 24(2):87-96.

[37] Yosef, T., Mengistu, U., Solomon, A., Mohammed, Y.K. \& Kefelegn, K. (2013) Camel and cattle population dynamics and livelihood diversification as a response to climate change in pastoral areas of Ethiopia. Livestock Research for Rural Development, 25(9).

[38] Zadeh, F., \& Murdoch, A.J. (2001) The effects of temperature and moisture on after-ripening of Cenchrus ciliaris seeds. Journal of arid environments, 49(4), 823-831. 